\title{
Kotuntersuchungen bei chronisch-entzündlichen Darmerkrankungen
}

Doris Bismarck

\section{Die Diagnosefindung bei Patienten mit chronischen Enteropathien ist oft nicht einfach. Im Folgenden werden Symptome besprochen und labordiagnostische Möglichkeiten der Kotuntersuchung vorgestellt, die zur Ursachenabklärung beitragen können.}

\section{Einleitung}

Klinisch manifestiert sich eine chronische Darmentzündung mit Erbrechen und Durchfall. Weitere Symptome sind abdominale Schmerzhaftigkeit, Darmgeräusche, Flatulenz, Gewichtsverlust sowie Inappetenz oder auch Polyphagie.

Chronisch-entzündliche Darmerkrankungen bei Hunden und Katzen können vielerlei Ursachen haben und eine diagnostische Herausforderung darstellen. Mögliche Ursachen sind Infektionen mit Protozoen, Parasiten oder pathogenen Bakterien. Eher selten kommen Mykobakterien und mykologische Erreger wie Histoplasma oder Algen wie Prototheken als Ursache in Betracht. Immer häufiger auftretend sind Futtermittelüberempfindlichkeiten. Darmentzündungen können auch sekundär infolge anderer primärer Darmerkrankungen wie einem Lymphom auftreten.

Chronische Darmentzündungen werden gern und viel zu häufig ohne Ausschluss anderer Ursachen mit dem Begriff Inflammatory Bowel Disease (IBD) assoziiert. Bei der IBD handelt es sich aber streng genommen um eine idiopathische chronische Darmentzündung, bei der die histopathologische Untersuchung von Darmbiopsien als Goldstandard zählt. Allerdings stellt dies eine sehr aufwendige und invasive Methode dar und sollte somit eines der letzten diagnostischen Mittel sein. Kotuntersuchungen dagegen gestalten sich einfach und sollten immer, wie auch andere diagnostische Schritte, vor der Verdachtsdiagnose IBD durchgeführt werden. Koprologische Untersuchungen können bereits erste Antworten auf die teils schwierige Ursachenfindung chronischer Enteropathien liefern.

\section{Kotprobendiagnostik}

Folgende diagnostische Schritte kommen in Betracht:

- parasitologische Kotuntersuchung

- kulturelle Kotuntersuchung

- Marker für Proteinverlust

- Entzündungsmarker

\section{Parasitologische Kotuntersuchung}

Die Kotuntersuchung auf Parasiten und Protozoen kann ein Nativpräparat, verschiedene Anreicherungsmethoden wie Flotations- und Sedimentationsverfahren, einen Enzyme-linked Immunosorbent Assay (ELISA), eine Polymerase-Kettenreaktion (PCR) und selten auch eine kulturelle Anzucht umfassen ( $\triangleright$ Tab. $\mathbf{1})$.

Ursächlich können einige Parasiten und Protozoen für eine chronische Darmentzündung sein:

- Giardien

- Tritrichomoas foetus (Katzen)

- Hakenwürmer (Ancylostoma und Uncinaria spp.)

- Spulwürmer (Toxocara spp. und Toxascaris leonina)

- Peitschenwürmer (Trichuris vulpis)

Merke

Eine Sammelkotprobe von 3 Tagen oder von 3 Defäkationen erhöht die Sensitivität der parasitologischen Untersuchung.

Auch bei negativer parasitologischer Untersuchung wird von einigen Autoren sicherheitshalber eine Therapie mit Fenbendazol empfohlen. Bei der parasitologischen Untersuchung ist nur ein positiver Befund beweisend, ein negativer Befund ist kein 100\%iger Ausschluss eines Befalls. Fenbendazol hat ein breites Wirkspektrum und ist gegen häufig im Darmtrakt vorkommende Nematoden, Giardien und einige Bandwurmarten wirksam. 
- Tab. 1 Nachweisverfahren ausgewählter Protozoen und Parasiten.

\begin{tabular}{|c|c|c|c|c|c|}
\hline Parasiten & $\begin{array}{l}\text { Nativ- } \\
\text { präparat }\end{array}$ & Kultur & $\begin{array}{l}\text { parasitologische } \\
\text { Untersuchung } \\
\text { (Flotation und } \\
\text { Sedimentation) }\end{array}$ & ELISA & PCR \\
\hline Giardia spp. & $(+)^{1}$ & - & + & + & +2 \\
\hline Tritrichomonas foetus & $(+)^{1}$ & $(+)^{3}$ & - & - & + \\
\hline Hakenwürmer (Ancylostoma \& Uncinaria) & - & - & + & - & - \\
\hline Spulwürmer (Tococara spp. \& Toxascaris leonina) & - & - & + & - & - \\
\hline Peitschenwürmer (Trichuris vulpis) & - & - & + & - & - \\
\hline
\end{tabular}

\section{Giardia duodenalis}

Die Trophozoiten, eines der beiden Protozoenstadien, heften sich im Dünndarm an das Mukosaepithel und verändern dessen Permeabilität. Giardieninfektionen verlaufen bei Hund und Katze oft symptomlos. Besonders bei Jungtieren kann aber eine intermittierende Diarrhoe gekennzeichnet durch voluminösen, dünnbreiigen bis wässrigen Kot mit Schleim- und teils auch mit Blutbeimengungen auftreten.

\section{Tritrichomoas foetus}

Der Erreger kolonisiert die Kolonmukosa von Katzen und führt zu chronischer Dickdarmdiarrhoe. Junge Katzen, die in einem Mehrkatzenhaushalt leben, sind besonders empfindlich. Bei persistierenden Infektionen kommt es zu einer intermittierenden Symptomatik, die besonders in Stresssituationen aufflammt. Weicher bis flüssiger Kot mit Schleim- oder Blutbeimengungen kann auftreten. Es zeigt sich eine erhöhte Kotabsatzfrequenz bis hin zur Kotinkontinenz.

\section{Hakenwürmer (Uncinaria und Ancylostoma)}

Bei Hunden werden die Hakenwürmer Ancylostoma caninum (eher wärmere Regionen) und Uncinaria stenocephala nachgewiesen. Katzen sind mit Ancylostoma tubaeforme und nur selten mit Uncinaria befallen. Hakenwürmer haben teils komplexe Entwicklungszyklen. Die Larven durchlaufen unter anderem im Magen und Duodenum verschiedene Reifestadien. Einige können zusätzlich in die Lunge und in andere Organe gelangen. Sie treten klinisch häufig bei Jungtieren in Erscheinung. Je nach Stärke des Befalls kann es beim Jungtier zu Durchfall, Blutverlust und möglicherweise zu einer ProteinverlustEnteropathie kommen.

\section{Spulwürmer (Toxocara spp. und Toxascaris leonina)}

Hund und Katze können vom Spulwurm Toxocara canis bzw. Toxocara cati befallen sein. Die Larven können die Darmwand durchdringen und ebenfalls in die Lunge und andere Organe wandern. Der Spulwurm führt bei Jungtieren zu schleimig, blutigem Durchfall abwechselnd mit
Obstipation, Erbrechen und bei hohem Befall zu einem umfangsvermehrten, schmerzhaften Abdomen, dem sogenannten Askaridenbauch. Toxascaris leonina befällt hauptsächlich den Dünndarm von Hund und Katze und führt, wenn überhaupt zu einer milderen Symptomatik.

\section{Peitschenwurm (Trichuris vulpis)}

Der Peitschenwurm kann Ursache einer akuten oder chronisch-intermittierenden Dickdarmdiarrhoe beim adulten oder juvenilen Hund sein. Die adulten Würmer dringen mit dem Vorderkörper in das Epithel der Zäkum- und Kolonmukosa und sorgen für eine Entzündung. Klinisch zeigen sich Durchfall mit Blut- und Schleimbeimengungen, Erbrechen, ein schmerzhaftes Abdomen und Gewichtsverlust. Bei massivem Befall kann es zu Anämie und Proteinverlust kommen. Allerdings sind auch symptomlose Trägertiere bekannt. Bei der Katze sind Peitschenwürmer eher selten.

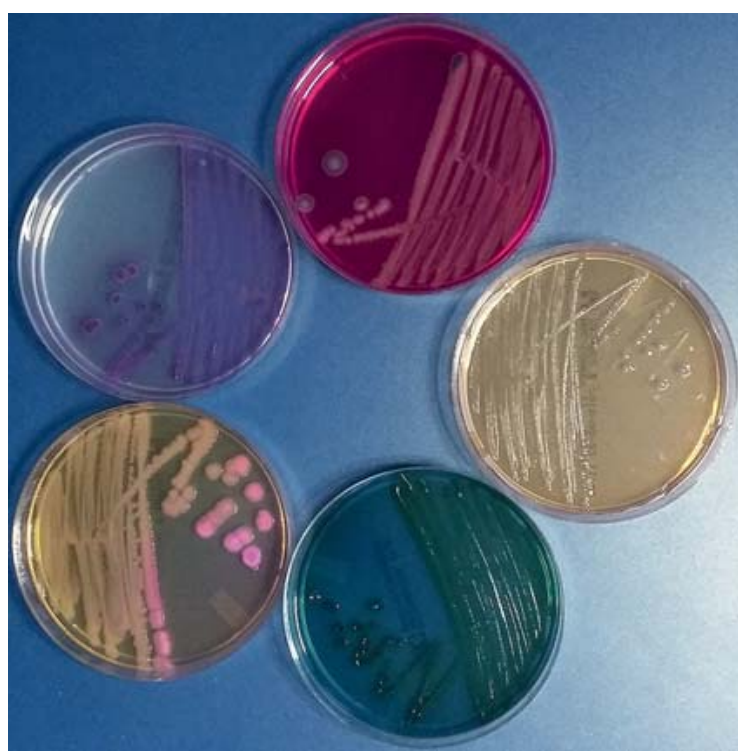

Abb. 1 Wachstum verschiedener Darmbakterien auf Selektivnährmedien. (C) Laboklin 


\section{Kulturelle Kotuntersuchung}

Mit einer kulturellen Kotuntersuchung ( $\bullet$ Abb. 1, Tab. 2) kann der Nachweis sogenannter darmpathogener Keime gelingen:

- Salmonellen

- Campylobacter spp.

- Clostridium perfringens und difficile

- Yersinia spp.

- enteropathogene Escherichia (E.) coli

Die Keime werden allerdings auch bei klinisch gesunden

Tieren nachgewiesen, weshalb sie für eine vorliegende
Entzündung nicht zwangsläufig verantwortlich sein müssen. Die Pathogenität der genannten Bakterien ist in der Fachliteratur umstritten. Es gibt Äußerungen, dass bisher keine veröffentlichte wissenschaftlich basierte Studie existiert, die belegt, dass Clostridium, Salmonella oder Campylobacter spp. chronische Enteritiden bei Hunden verursachen.

Für die Anzucht darmpathogener Bakterien bedarf es oft einiger Spezialnährböden ( $\bullet$ Abb. 1 ) und besonderer Kulturbedingen. 


\section{Merke}

Ein spezieller Keimverdacht sollte bei der diagnostischen Abklärung der Kotprobe mit angegeben werden.

\section{Salmonellen}

Eine Salmonelleninfektion kann sich klinisch unter anderem durch Erbrechen, Durchfall, Fieber, Anorexie und Lethargie äußern. Ca. $1 / 3$ klinisch gesunder Hunde können asymptomatische Salmonellenausscheider sein. Die Erreger können in den regionalen Lymphknoten persistieren, sodass betroffene Tiere über mehrere Wochen Ausscheider bleiben. Eine intermittierende Ausscheidung ist möglich.

\section{Campylobacter}

Wenn bei einer Campylobacter-Infektion Durchfall auftritt, kann dieser von gelegentlich weichem Kot bis zur hochgradig wässrigen und auch hämorrhagischen Diarrhoe teils mit Schleimbeimengungen reichen. Erbrechen und Anorexie können ebenfalls klinische Symptome sein.

\section{Clostridien}

Clostridien gehören zur physiologischen Darmflora. Clostridium perfringens und Clostridium difficile wurden bei Enteritiden isoliert, in seltenen Fällen können sie durch Toxinproduktion zu Enterotoxämien führen. Wenn eine Clostridieninfektion klinisch in Erscheinung tritt, kann dies milde Symptome bis hin zu akutem und auch chronischem Dick- oder Dünndarmdurchfall mit Blut- und Schleimbeimengungen beinhalten. Teilweise sind Erbrechen und Tenesmus zu beobachten. Clostridium perfringens Enterotoxin und Clostridium difficile Toxin A und B können auch bei klinisch gesunden Hunden gefunden werden. Die Entwicklung einer Diarrhoe ist eventuell konzentrationsabhängig.

\section{Yersinien}

Yersinia enterocolitica und Yersinia pseudotuberculosis können als Darmbewohner bei gesunden Tieren vorkommen, aber auch zu chronischer Kolitis mit blutigem, schleimigen Durchfall führen. Eine erhöhte Kotabsatzfrequenz mit Tenesmus kann bei klinischer Symptomatik ebenfalls beobachtet werden.

\section{E. coli}

Der Erreger bildet einen wichtigen Bestandteil der physiologischen Darmflora. Bei einer Schwächung der lokalen oder systemischen Abwehr können pathogene E. coli Durchfall und Erbrechen verursachen. In schweren Fällen ist auch eine E.-coli-Sepsis möglich. Enteropathogene E. coli besitzen unterschiedlichste Virulenzfaktoren wie Adhäsine, Kapselpolysaccharide und Endotoxine.

\section{Marker für gastrointestinalen Proteinverlust}

Bei einer Proteinverlust-Enteropathie (Protein-losing enteropathy, PLE) gehen Proteine über die entzündete Darmmukosa verloren. Die Erkrankung sollte stets bei
- Tab. 2 Nachweismethoden ausgewählter Endoparasiten.

\begin{tabular}{|c|c|c|c|}
\hline $\begin{array}{l}\text { darmpathogene } \\
\text { Bakterien }\end{array}$ & Kultur & ELISA & $\mathrm{PCR}^{4}$ \\
\hline Salmonellen & + & - & + \\
\hline Campylobacter & +1 & - & + \\
\hline Clostridien & $t^{2}$ & - & - \\
\hline Clostridientoxine & - & + & - \\
\hline Yersinien & +3 & - & + \\
\hline E. coli & + & - & - \\
\hline Pathogenitätsfaktoren & - & - & + \\
\hline \multicolumn{4}{|c|}{$\begin{array}{l}{ }^{1} \text { Campylobacter benötigt mikroaerophile Wachstumsbedin- } \\
\text { gungen. }\end{array}$} \\
\hline \multicolumn{4}{|c|}{$\begin{array}{l}{ }^{2} \text { Clostridien benötigen anaerobe Wachstumsbedingungen, } \\
\text { die Bestimmung der Gesamtheit der gasbildenden Bakte- } \\
\text { rien ist auch im Hochschichtagar möglich. }\end{array}$} \\
\hline \multicolumn{4}{|c|}{$\begin{array}{l}{ }^{3} \text { Yersinien benötigen eine spezielle Kälteanreicherung, die } \\
\text { bis zu } 3 \text { Wochen dauern kann. }\end{array}$} \\
\hline \multicolumn{4}{|c|}{$\begin{array}{l}{ }^{4} \text { Die Anfertigung eines Antibiogramms ist bei einem PCR- } \\
\text { Nachweis nicht möglich. }\end{array}$} \\
\hline
\end{tabular}

Tieren mit Hypoproteinämie und gastrointestinalen Symptomen in Betracht gezogen werden. Die Bestimmung von $\alpha$-1-Antitrypsin ( $\alpha$-1-Proteinase-Inhibitor) kann diagnostisch wertvoll sein.

Hierbei handelt es sich um einen im Serum vorkommenden Proteinase-Inhibitor mit einer ähnlichen Molekularmasse wie Albumin. Kommt es zum erhöhten Verlust von Albumin über die Darmwand, geht auch vermehrt $\alpha-1$-Antitrypsin ins Darmlumen über. Allerdings wird dieses Protein aufgrund seiner Eigenschaft eines ProteinaseInhibitors nicht wie Albumin von endogenen oder bakteriellen Proteinasen abgebaut. Es wird in der gleichen Menge mit dem Kot ausgeschieden wie es über die erkrankte Darmwand verloren geht.

$\alpha$-1-Antitrypsin sollte aus 3 aufeinanderfolgenden Defäkationen bestimmt werden, da hohe individuelle Schwankungen auftreten.

\section{Marker für gastrointestinale Entzündungen}

Ein relativ neuer Marker in der Tiermedizin ist Calprotectin. Es handelt sich um ein Kalzium-bindendes Protein mit antimikrobiellen Eigenschaften, das als Marker für neutrophile Entzündungen angesehen wird. Anfängliche Studien haben gezeigt, dass es in Fäzes von Hunden mit chronischen Enteropathien erhöht ist. Physiologischerweise ist Calprotectin beispielsweise auch bei Welpen erhöht. Zu Calprotectin bei Katzen gibt es noch keine bekannten Studien. 


\section{Fazit}

Kotuntersuchungen können Aufschluss über Ursachen oder zusätzliche Hinweise bei der Abklärung chronischer Enteropathien geben. Sie sollten bei der diagnostischen Aufarbeitung stets vor invasiveren Methoden wie der histopathologischen Untersuchung durchgeführt werden.

Korrespondenzadresse

\section{Dr. Doris Bismarck}

Laboklin GmbH \& Co KG

Steubenstraße 4

97688 Bad Kissingen

bismarck@laboklin.com

Literatur

[1] Deplazes P, Eckert J, von Samson-Himmelstjerna G, Zahner H. Lehrbuch der Parasitologie für die Tiermedizin 3. Aufl. Stuttgart: Enke; 2013

[2] Grellet A, Heilmann RM, Lecoindre P et al. Fecal calprotectin concentrations in adult dogs with chronic diarrhea. Am J Vet Res 2013; 74: 706-711

[3] Kohn B, Schwarz G, Hrsg. Praktikum der Hundeklinik. 12. Aufl. Stuttgart: Enke; 2012

[4] Steiner JM. Review of commonly used clinical pathology parameters for general gastrointestinal disease with emphasis on small animals. Toxicol Pathol 2014; 42: 189-194

[5] Steiner JM. Gastroenterologie bei Hund und Katze. Hannover: Schlütersche; 2011

[6] Westermarck E. Chronic diarrhea in dogs: what do we actually know about it? Topics Companion Anim Med 2016; 31: 78-84

Bibliografie

DOI https://doi.org/10.1055/a-0579-1050

Kleintier konkret 2018; 21: 35-39

(c) Georg Thieme Verlag KG Stuttgart · New York ISSN 1434-9132 